\title{
Menopause: A Reproductive Health Issue
}

\author{
Shipra Nagar* \\ College of Community Science, Central Agricultural, University, Tura, Meghalaya, India \\ *Corresponding author
}

Keywords

Menopause,

Reproductive

health, Middle age

Article Info

Accepted:

16 November 2020

Available Online:

10 December 2020

\begin{abstract}
A B S T R A C T
Menopause is one of the most significant events in lives of middle aged women. It is an

emerging reproductive health issues which has received less attention in past but with advent of health awareness, improved socio-economic conditions and better educational status, it is now becoming a concern for most of the women in their 40s and 50s. Women spend almost one third of their lives in the post-menopausal states and thus becomes a major concern for the policy makers in terms of their reproductive health. Women tend to suffer not only from the health hazards of their reproductive life span but are also subjected to the stresses coming to them after the reproductive period comes to an end. The average age at menopause is said to occur between 40 and 50s but may differ considerably within the country varying from region to region. The changes occurring to the women during this stage are not only physical but also emotional which requires support from not only the partner but also from children and other family members. This article presents an overview of menopausal changes occurring to women and ways to support.
\end{abstract}

\section{Introduction}

The concept of reproductive health has occupied a central place in the field of health, illness and medicine after the International Conference on Population and Development (ICPD) was held in Cairo in 1994. It was during this conference that the reproductive health needs of women were felt essential. According to United Nations Reproductive health can be defined as "a state of complete physical, mental and social well-being and not merely the absence of disease or infirmity in all matters relating to the reproductive system and its processes" (United Nations, 1994). Many researchers and policymakers have emphasized that the real reproductive health framework should go beyond the narrow confines of family planning to encompass all aspects of human sexuality and reproductive health needs during the various stages of women's lives.

The above definition laid emphasis on the importance given to all the concepts of reproductive health from menarche to menopause. The concept of menopause got its importance in 1981 when a report of the World Health Organization, Scientific Group, based on its meeting on Research on the Menopause held in Geneva during December 1980 reported that there were virtually no data 
on the age distribution of the menopause and no information on its socio-cultural significance in the developing countries. Further the Scientific Group made some specific recommendations as WHO sponsored research should be undertaken to determine the impact on health service needs of the rapidly increasing numbers of postmenopausal women in developing countries; uniform terminology should be adopted by health care workers with regard to the menopause; uniform endocrine standards should be developed which can be applied to the description of peri and postmenopausal conditions and diseases; and descriptive epidemiological studies of the age at menopause should be performed in a variety of settings.

Menopause is a physiological event in a woman's life and is caused by aging of ovaries which leads to decline in the production of ovarian gonadotrophins, estrogen and progesterone. The deficiency of these hormones elicits various somatic, vasomotor, sexual and psychological symptoms that impair overall quality of life of women. It is said that the average woman is post menopausal for one-third of her life and thus the incidence of certain conditions like coronary artery disease, diabetes, breast cancer, cervical cancer and osteoporosis increases after menopause.

Menopause is perhaps the most striking event occurring during the middle age in women and represents the end of woman's reproductive life. The age at which the natural menopause (NM) occurs is between the age of 45 and 55 years worldwide (WHO 1996). With increase in life expectancy of women and with better medical facilities the percentage of women living beyond $50 \mathrm{~s}$ is increasing worldwide. In 1990, there were about 467 million postmenopausal women worldwide and this figure is expected to rise to 1200 million by 2030 and out of these, $76 \%$ will be living in the developing countries. According to the 2011 census of India, there were about 96 million women aged $45 \mathrm{yr}$ and above which is expected to increase to 401 million in 2026. It is said that by 2030 , the proportion of post-menopausal women living in industrialized regions will decline to $24 \%$ and about $76 \%$ will be living in developing countries (Kriplani and Banerjee, 2005).

As the life expectancy at age 45 is thirty years, women in India, on average, could spend approximately $30 \mathrm{yr}$ in postmenopausal stage of life. Since the postmenopausal years are associated with health risks such as hypertension, heart disease, osteoporosis and decline in the overall quality of life (Aaron et al., 2002 and Nagata et al., 1998), this group will present profound future challenges to public healthcare provision. Thus, health policies in the country should also focus on this age group of women, who are beyond 50s, as the vulnerability of getting multiple illnesses like osteoporosis, heart diseases, etc. generally increases.

\section{Menopause: A characteristic feature of Middle age}

Middle age or mid-adulthood is the period that starts roughly when an individual is in 40 s and ends at the time when old age begins and is thus characterized by a general decline in physical fitness. From the view of developmental psychology, the major developmental task of middle age centers around learning to accept the physiological changes and make adjustments accordingly (Brooks and Kirsh, 1984). This task is important for a woman due to occurrence of menopause, which brings the reproductive capacity of a woman, to bear children, to a halt and in turn have a significant effect on women's physical and mental health 
One of the features of middle age, important in a woman's life, is the end of her childbearing age through menopause. Menopause is a normal but one of the major transitional periods in the life of every woman. The word 'Menopause' is derived from the Greek word 'menos' means month and 'pause' means to stop - which refers to the 'last menstrual period' and is generally considered to have occurred after one year of amenorrhoea. According to he World Health Organization, menopause is the cessation of ovarian function which is represented by absence of menses for at least 12 months (WHO 1996). Once the body of woman has completed the changes, and she has not had periods for 12 months in a row, she is said to have passed through menopause (Margolese, 2003).

The menopause is emerging as an issue owing to rapid globalization, urbanization, awareness and increased longevity in urban middle-aged Indian women, who are evolving as a homogeneous group. Improved economic conditions and education may cause the attitude of rural working women to be more positive towards the menopause. Menopause is a reproductive milestone in a woman's life. It brings a woman acutely face to face with the reality of aging. It is a complex transition involving biological, psychological, sociological and cultural variables. Menopause should be viewed as a process and not an event, as something which is shaped by historical factors and environmental and cultural context, and by each individual's personal history, both biological and social.

The whole process of transition into menopause can be divided into different phases as: Premenopause, Perimenopause and Postmenopause.

Pre-menopause: menstrual period in the past 3 months and no decreased predictability.
Peri-menopause: menstrual bleeding in the past 12 months but not in the past 3 months.

Post-menopause: amenorrhea for the past 12 months and no hysterectomy.

\section{Age at Menopause}

The exact age at which menopause occurs varies from population to population. It is generally stated that the average age at menopause falls between 40 to 50 years, although the spread is from the 30 s to late 50s. In most developed countries, this event occurs around the age of 50 years (WHO 1996). But in developing countries like India there has been a trend in advancement of age at menopause (NFHS 2005-2006). Thus, along with increase in life expectancy growing number of these women can expect to live for several decades after menopause.

In India, due to vast cultural and regional differences, average age at menopause also varies as seen from different research studies covering different regions and states of the country. One of the earliest studies done in the country was that of Randhawa et al., (1987) done on Himachali women which suggested the average age at 43.55 years but in the following years till now the average age centers around 46 to 48 years for an average Indian women. Some of the past researches related to the age at menopause of Indian women have been presented in Table 1 .

Factors that affect age at menopause may have important clinical implications because early menopause is associated with an increased risk of cardiovascular disease and osteoporosis, whereas delayed menopause has been associated with increased risk of breast cancer and endometrial cancer (Hidayat et al., 1999). These associations may result from the direct effect of menstrual function (or cessation of function) and the related 
hormone changes, or may be an indirect result of the other factors that are associated with age at menopause. The relationship of menopausal age with the risk factors for such medical conditions makes age at menopause an important epidemiological issue.

\section{Changes occurring during menopause}

During the period of menopause, the body of a woman undergoes many changes, both internal and external. Change in the secretion of the endocrine hormones brings about more symptoms. Even though menopause is a physiological process, menopausal symptoms may be multi-factorial. Genetic factors, nutrition, socio-demographic factors, lifestyle, cultural factors, awareness and understanding of health issue, attitude toward health and access to health care services can influence the type, frequency and severity of symptoms (Ganitha et al., 2017).

The changes in the body include the hormonal changes and the changes in the female reproductive system.

\section{Hormonal changes}

Until menopause, a woman's ovaries periodically release estrogen during the monthly menstrual cycle. But as a woman reaches menopause, her ovaries stop producing a monthly ovum and secrete a smaller supply of estrogen. These hormonal changes mean that she will no longer be able to conceive and bear children, and that she might experience some uncomfortable physical symptoms as her body adjust to the new hormonal balance.

Female sex hormones estrogen and progesterone are produced in sub critical quantities for a short time after menopause, but over a few years fall almost to zero. These hormones are important for keeping the vagina and uterus healthy as well as for normal menstrual cycles and for successful pregnancy. Estrogen also helps to keep bones healthy. It helps women to keep good cholesterol levels in their blood. Levels of two other female hormones, progesterone and luteinizing hormone ( $\mathrm{LH})$, also rise and fall during your menstrual cycle. Progesterone levels drop after menopause, and LH levels go up. During menopausal transition there is a lot of fluctuation in these hormone levels and thus women may experience many symptoms and conditions. Some of the important and common symptoms women can experience during menopausal transition are changes in periods, hot flashes and night sweats, problems with vagina and bladder, changes in sexual desire, sleep problems, mood changes/swings, and changes in the body.

The symptoms during menopause are due to endocrine imbalance, i.e. reduced levels of estrogen and rise in pituitary gonadotropins (Mukherjee et al., 1996). Hormonal imbalances during menopause and after menopause may affect the general health and outlook of some women.

\section{Difficulties and Problems faced}

There are a number of physical and psychological symptoms of menopause brought about by estrogen deficiency. Commonly reported symptoms in different research studies include hot flushes, night sweats, sleep disturbances, urinary frequency, vaginal dryness, poor memory, anxiety and depression (Aaron et al., 2002 and Sharma et al., 2007). The most common symptoms are the somatic and the vasomotor symptoms which include hot flushes, profuse perspirations, etc. the various physical symptoms which are experienced by women during menopause are shown in the below figure: 


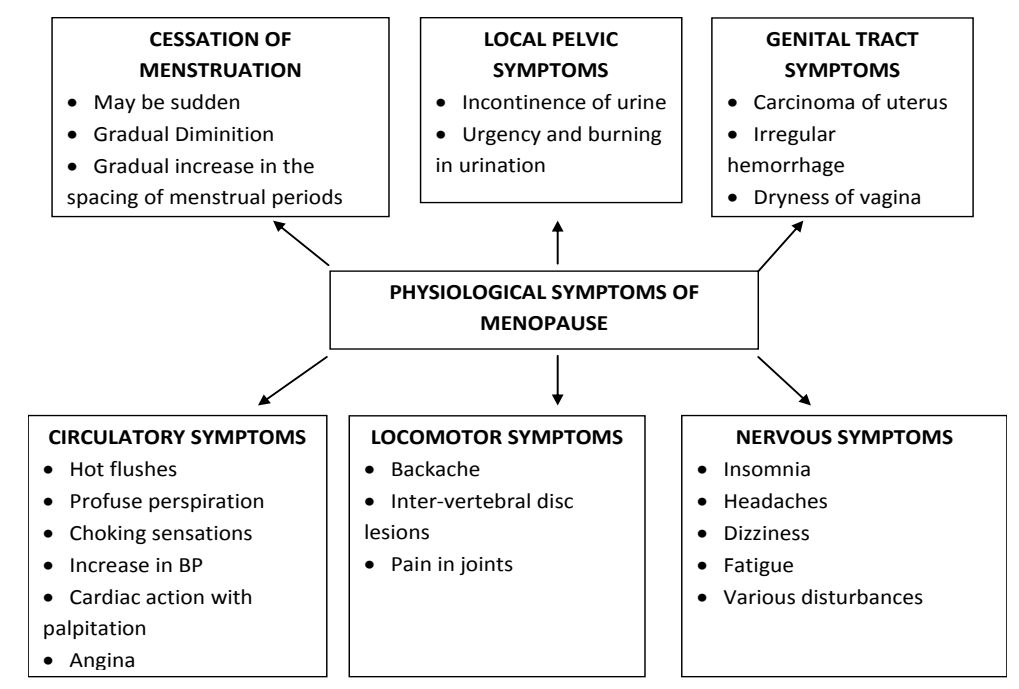

\section{Irregular vaginal bleeding}

Irregular vaginal bleeding may occur during menopause which may include excessive or minimal bleeding. Menstrual periods (menses) may occur more frequently (meaning the cycle shortens in duration), or they may get farther and farther apart (meaning the cycle lengthens in duration) before stopping.

\section{Hot flashes and night sweats}

A hot flash is a feeling of warmth that spreads over the body and is often most pronounced in the head and chest. A hot flash is sometimes associated with flushing and is sometimes followed by perspiration. Hot flashes are common among women undergoing menopause and ususlly last from 30 seconds to several minutes. Sometimes hot flashes are accompanied by night sweats (episodes of drenching sweats at nighttime). This may lead to awakening and difficulty falling asleep again, resulting in unrefreshing sleep and daytime tiredness.

\section{Vaginal symptoms}

Vaginal symptoms occur as a result of the lining tissues of the vagina becoming thinner, drier, and less elastic as estrogen levels fall. Symptoms may include vaginal dryness, itching, or irritation and/or pain with sexual intercourse (dyspareunia). The vaginal changes also lead to an increased risk of vaginal infections.

\section{Urinary symptoms}

The lining of the urethra (the transport tube leading from the bladder to discharge urine outside the body) also undergoes changes similar to the tissues of the vagina, and becomes drier, thinner, and less elastic with declining estrogen levels. This can lead to an increased risk of urinary tract infection, feeling the need to urinate more frequently, or leakage of urine (urinary incontinence). The incontinence can result from a strong, sudden urge to urinate or may occur during straining when coughing, laughing, or lifting heavy objects.

\section{Emotional and cognitive symptoms}

Women in perimenopause often report a variety of thinking (cognitive) and/or emotional symptoms, including fatigue, memory problems, irritability, and rapid changes in mood. 


\section{Other physical changes}

Many women report some degree of weight gain along with menopause. The distribution of body fat may change, with body fat being deposited more in the waist and abdominal area than in the hips and thighs. Changes in skin texture, including wrinkles, may develop along with worsening of adult acne in those affected by this condition. After menopause, many women have been found to experience genital and /or urinary troubles, prevalence of hypertension and coronary heart disease became high, osteoporosis and increased incidence of bone fractures are found, lower back ache found to be the major problem with fatigue, decreased memory, vaginal dryness, insomnia, loss of libido, dry skin and depression.

The recognition of advancing years and impending old age comes rather recently. The women notice the difference in their appearance, fear of cessation of the biological function of maturity and with the decrease in feminine attractiveness dread the possible loss of men's attention and affection. All these factors leads to the occurrence of symptoms as mood swings (depression), anxiety with tension, irritability, nervousness, lack of energy, difficulty in concentration in women.

Table.1 Average age at menopause of Indian women over the years

\begin{tabular}{|c|c|c|c|}
\hline SI.No & Authors & Year & Mean age at menopause \\
\hline 1. & Randhawa et al., & 1987 & 43.55 \\
\hline 2. & Mukherjee et al., & 1996 & 48.23 \\
\hline 3. & Nagar and Dave & 2005 & 44.59 \\
\hline 4. & Kriplani and Banerjee & 2005 & 48.0 \\
\hline 5. & Kaulagekar & 2006 & 47.6 \\
\hline 6. & Sharma et al., & 2007 & 47.35 \\
\hline 7. & Bairy et al., & 2009 & 48.7 \\
\hline 8. & Mahajan et al., & 2012 & 44.54 \\
\hline 9. & Madhukumar et al., & 2012 & 49.33 \\
\hline 10. & Madhu Kumar & 2012 & 49.7 \\
\hline 11. & Borker et al., & 2013 & 48.26 \\
\hline 12. & Sarkar et al., & 2014 & 45.3 \\
\hline 13. & Singh and Pradhan & 2014 & 46.24 \\
\hline 14. & Dasgupta et al., & 2015 & 41.17 \\
\hline 15. & Satpathy & 2016 & 44.82 \\
\hline 16. & Ahuja & 2016 & 46.2 \\
\hline 17. & Ganitha et al., & 2017 & 45.75 \\
\hline 18. & Jadhav and Bavaskar & 2017 & 45.0 \\
\hline 19. & Jeyasheela et al., & 2018 & 48.13 \\
\hline
\end{tabular}

\section{Osteoporosis}

Osteoporosis is a condition in which bones become very weak and can break easily. Since estrogen plays an important part in building new bone, the decline in estrogen starting with menopause leaves many older women at risk for developing osteoporosis. Osteoporosis is a serious risk for Indian women. Osteopenia, or low bone mineral density (BMD) usually means a greater chance of developing osteoporosis. 
Approximately $35-40 \%$ of Indian women aged 40 -65 suffer from osteopenia and they tend to have a low bone density due to lack of sufficient calcium intake and adequate exercise.

Risk factors, things that increase the chance of developing a health problem, include:

a family history of osteoporosis

a broken bone while an adult

surgery to remove both ovaries before natural menopause

early menopause

insufficient calcium throughout life

extended bed rest

smoking

long-term heavy drinking

using certain medicines, such as glucocorticoids or some anticonvulsants, for long periods of time

being thin or having a small body frame

\section{Cardiovascular diseases}

Common cardiovascular diseases include a narrowing and hardening of the arteries (atherosclerosis), chest pain that happens when not enough blood is getting to the heart (angina), heart attack, and stroke. High blood pressure (hypertension) is a strong risk factor for cardiovascular disease and stroke.

\section{Effect on spousal relationship and other social relations}

Menopause brings a radical change in the relationship of man and woman. Most of the women show lack of interest in sexual activities and may become withdrawn. With menopause there is lack of necessary and essential hormones - estrogen and progesterone - in the body. As a result of it, there will be decrease in the flow of blood in the vagina. Because of this, walls of vagina will become thinner and they will loose their elasticity. These factors cause discomfort while having sex. There may be irritation and infection too causing serious aversion to sex. Thus menopause, all over the world, is believed to be a cause in the decrease in sexual activity.

In conclusion the mid-life can be an exciting period in our lives. It is a time of changephysical changes, of course, but possibly emotional and social adjustments, too. It might also be a time of acceptance — of these changes and of our evolving roles in life. This is also a time of opportunity and promise - a chance to make healthy changes in our lifestyle that will allow us to get the most out of the rest of our life. Women and men need to educate their partners about the changes that occur in their bodies as well as those they experience. They can build mutual support by keeping one another informed. Counseling of couples can be very valuable for partners in mid-life. Other family members, relatives and friends can provide important support during this crucial stage of menopause. More than anything else, preparing oneself for perimenopause and menopause psychologically and emotionally works out more effectively. One should eat healthy diet, which is low in fat and cholesterol, high in fibre and well balanced with vitamins and minerals, mainly calcium. Fruits, vegetables, and whole grains should be included in the daily diet. Regular general check-up including blood pressure and blood sugar, checking cholesterol is very much essential. Regular pelvic and breast examinations are to be done. With the increasing life expectancy today's women will live a third of her life after menopause. Health education and planning ahead for challenges can make this period as one of the most rewarding and enriching time of her life.

\section{References} $\begin{array}{rr}\text { Aaron R, Muliyil J, Abraham S. } & 2002 . \\ \text { Medico-social } & \text { dimensions }\end{array}$ 
menopause: A cross- sectional study from rural south India. National Medical Journal of India, 15: 14-17.

Ahuja, M. 2016. Age of menopause and determinants of menopause age: A PAN India Survey by IMS. Journal of Midlife Health, 7: 126-131.

Bairy, L. Adiga, S. Bhat, P. and Bhat, R. 2009. Prevalence of menopausal symptoms and quality of life after menopause in women from South India. Australian and New Zealand Journal of Obstetrics and Gynaecology, 49: 106109.

Becker, D. Comranz, J. and Pines, A. 2001. Psychological distress around menopause. Psychosomatics, 42: 252257.

Borker SA, Venugopalan PP, Bhat SN. 2013. Study of menopausal symptoms, and perceptions about menopause among women at a rural community in Kerala. Journal of Midlife Health, 4:182-7.

Brooks Gunn, J. and Kirsh, B. 1984. Life events and the boundaries of midlife for women. In G.K. Baruch and J. Brooks Gunn (Eds.) Women in midlife (pp1130), New York:Plenum.

Dasgupta, D. Karar, P. Ray, S. and Ganguly, N. 2015. Menopausal symptoms and its correlates: A study in tribe and caste population of East India. Current Gerontology and Geriatrics Research. Article ID 984767, 7 pages, http://dx.doi.org/10.1155/2015/984767

Ganitha, G. Premalatha, P.B. and Kanan, I. 2017. A study of the age of menopause and menopausal symptoms among women in a rural area of Tamil Nadu, India. Journal of South Asian Federation of Menopause Societies, 5(2): 87-91.

Hidayat, N.M. Sharaf, S.A. Aref, S.R. Tawfik, T.A. and Moubarak, I. 1999. Correlates of age at natural menopause: A community based study in
Alexandria. East Mediterranean Health Journal, 5:307-319.

Indian Institute of Population Sciences (IIPS) and ORCMacro, National Family and Health Survey-3, 2005-2006, IIPS, Mumbai, India, 2007

Jadhav, A. and Bavaskar, Y. 2017. An epidemiological health problems in women living in an urban area of Mumbai, Maharashtra. International Journal of Community Medicine and Public Health, 4(9): 3088-93.

Jeyasheela, K. Ebenezer, E.D. Londhe, V. Paul, T.V. Yadav, B. and Kekre, A.N. 2018. Prevalence of metabolic syndrome among postmenopausal women in South India. International Journal of Reproduction, Contraception, Obstetrics and Gynecology, 7 (6): 2364 2370

Kaulagekar, A. 2006. Age at menopause and menopausal symptoms among urban women in Pune, Maharashtra. Journal of Obstetrics and Gynaecology of India, 61(3): 323-326.

Kriplani A, Banerjee K. 2005. An overview of age of onset of menopause in Northern India. Maturitas, 52:199-204.

Madhukumar, S. Gaikwad, V. and Sudeepa, D. 2012. A Community Based Study on perceptions about menopausal symptoms and quality of life of post menopausal women in Bangalore rural. International Journal of Health Science and Research, 2(3):49-56.

Mahajan, N. Aggarwal, M. and Amrita Bagga. 2012. Health issues of menopausal women in North India. Journal of Midlife and Health, 3(2):8487.

Mukherjee, P. Nayak, A.and Mullick, J. 1996. Physical and psychological symptoms comparative study of effect of oestrogen replacement and psychotropics in menopausal distress. Journal of Obstetrics and Gynaecology of India, 
46(2): 244-249.

Nagar S, Dave P. 2005 Perceptions of women towards physiological problems faced at menopause. The Anthropoligist, 7(3):173-5.

Nagata C, Takatsuka N, Inaba S, Kawakami N, Shimizu H. 1998. Association of diet and other lifestyle with onset of menopause in Japanese women. Maturitas. 29:105-13.

Population Projections for India and States 2001-2026. New Delhi: Government of India; 2006. Office of the Registrar General and Census Commissioner.

Randhawa, I. Premi, H.K. and Gupta, T. 1987. The age at menopause in the women of Himachal Pradesh, and the factors affecting menopause. Indian Journal of Public Health, 31:40-44.

Sagar A. Borker, P. P. Venugopalan, and Shruthi N. Bhat. 2013. Study of menopausal symptoms, and perceptions about menopause among women at a rural community in Kerala J Midlife Health, 4(3): 182-187.

Satpathy, M. 2016. A study on Age at menopause, menopausal symptoms and problems among Urban women from Western Odisha, India. International Journal of Scientific and Research Publication, 6(3): 422-427
Sharma, S. Tandon, V.R. and Mahajan, A. 2007. Menopausal Symptoms in Urban Women. JK Science, 9(1):13-17.

Singh, A. and Pradhan, S.K. 2014. Menopausal symptoms of postmenopausal women in a rural community of Delhi, India: A Crosssectional study. Journal of Midlife and Health 5(2): 62-67.

United Nations. 1994. Programme of action of the 1994 International Conference on Population and Development (A/CONF.171/13). Reprinted in Population and Development Review 21(1) and 21(2).

World Health Organization Scientific Group. 1996. Research on Menopause in 1990s WHO Technical Series No. 866. Geneva, Switzerland: WHO.

Zurayck, H. 1994. Population and health International Conference on Population and Development ICPD 1994. IUSSP, Liege.

ttp://www.nia.nih.gov/health/publication/men opause-time-change/staying-healthy

http://www.menopause.org/forwomen/sexual-health-menopauseonline/effective-treatments-for-sexualproblems

\section{How to cite this article:}

Shipra Nagar. 2020. Menopause: A Reproductive Health Issue. Int.J.Curr.Microbiol.App.Sci. 9(12): 2411-2419. doi: https://doi.org/10.20546/ijcmas.2020.912.286 